\title{
HARMONIC MAPS WITH FINITE TOTAL ENERGY
}

\author{
SHIU-YUEN CHENG, LUEN-FAI TAM, AND TOM Y.-H. WAN
}

(Communicated by Peter Li )

\begin{abstract}
We will give a criteria for a nonnegative subharmonic function with finite energy on a complete manifold to be bounded. Using this we will prove that if on a complete noncompact Riemannian manifold $M$, every harmonic function with finite energy is bounded, then every harmonic map with finite total energy from $M$ into a Cartan-Hadamard manifold must also have bounded image. No assumption on the curvature of $M$ is required. As a consequence, we will generalize some of the uniqueness results on homotopic harmonic maps by Schoen and Yau.
\end{abstract}

In this note, we will study harmonic maps with finite total energy on noncompact manifolds. The first main result we will prove is the following (Theorem 3.1): if $M$ is a complete noncompact manifold such that every harmonic function on $M$ with finite Dirichlet integral is bounded, then every harmonic map with finite energy from $M$ into a Cartan-Hadamard manifold must also be bounded. Results similar to this have been proved by many authors. It was proved by Yau $[\mathrm{Y}]$ that there is no nonconstant bounded harmonic function on a complete noncompact manifold with nonnegative Ricci curvature. The first author $[\mathrm{Cg}]$ proved that this is also true for harmonic maps from such a manifold into a Cartan-Hadamard manifold. This result was later generalized by Choi [Ci] and Kendall [Ke]. In particular, Kendall [Ke] proved that if $M$ supports no nonconstant bounded harmonic function and is stochastically complete, then $M$ also supports no nonconstant bounded harmonic map into a Cartan-Hadamard manifold. We should mention that even though the result in $[\mathrm{Ke}]$ is more general, however, in the proofs of $[\mathrm{Cg}]$ and $[\mathrm{Ci}]$, useful estimates on the energy density of a harmonic map were obtained. Recently, Sung, Wang and the second author [S-T-W] proved that if every bounded harmonic function on $M$ is asymptotically constant near infinity at each unbounded component of the complement of some compact set, then every bounded harmonic map from $M$ into a Cartan-Hadamard manifold also has this property. Using those results on bounded harmonic maps, one can obtain a Liouville type theorem for harmonic maps with finite total energy. For example, we show that (Theorem 3.2) if $M$ supports no nonconstant bounded harmonic function, then every harmonic map with finite total energy from $M$ into a Cartan-Hadamard manifold must be constant. This can be considered as a generalization of the result in [S-Y 1] which says

Received by the editors July 28, 1994.

1991 Mathematics Subject Classification. Primary 53C99; Secondary 31C05, 58E20.

The first and the third authors are partially supported by Earmarked Grant, Hong Kong, and the second author is partially supported by NSF grant \#DMS9300422. 
that if $M$ has nonnegative Ricci curvature, then there is no nonconstant harmonic map with finite total energy from $M$ into a complete manifold with nonpositive curvature.

We will also generalize some uniqueness results on homotopic harmonic maps on noncompact manifolds by Schoen and Yau [S-Y 2]. In [S-Y 2], it was proved that if $M$ has finite volume and $N$ has strictly negative sectional curvature and if $u$ is a harmonic map from $M$ into $N$ with finite total energy, then there is no other harmonic map with finite total energy which is homotopic to $u$ unless $u(M)$ is contained in a geodesic of $N$. It was also proved in [S-Y 2] that if $M$ has finite volume then for any two homotopic harmonic maps $u$ and $v$ with finite energy into a manifold with nonpositive sectional curvature, there exists a homotopy from $u$ to $v$ via geodesics. We will prove that these two results are still true (Theorems 3.5 and 3.6) by only assuming that $M$ has no positive Green's function. Note that a complete manifold which supports a positive Green's function must have infinite volume (see [V] or [L-T 1]). The main results in this note are quite general, and in most cases, there is no curvature assumption on the domain manifolds.

\section{Subharmonic functions With finite Dirichlet integral}

We begin with the following lemma.

Lemma 1.1. Let $M$ be a complete noncompact manifold. Let $f$ be a smooth function on $M$ which has finite Dirichlet integral and is subharmonic outside $B_{p}\left(r_{0}\right)$ for

some $r_{0} \geq 0$ and some point $p$. Suppose there is $r_{1}>r_{0}$ such that $\int_{\partial B_{p}\left(r_{1}\right)} \frac{\partial f}{\partial r}>0$. For all $R>r_{1}$, let $u_{R}$ be the solution of

$$
\Delta u_{R}=-\Delta f \text { on } B_{p}(R)
$$

and let $u_{R}=0$ on $\partial B_{p}(R)$. Then $u_{R}(p)$ are uniformly bounded from above.

Proof. Multiplying (1.1) by $u_{R}$ and integrating by parts, it is easy to see that

$$
\int_{B_{p}(R)}\left|\nabla u_{R}\right|^{2} \leq \int_{M}|\nabla f|^{2},
$$

for all $R$. Hence, by the Bochner formula and the mean value inequality (see for example, [L-T 2]), for all $r>0$, there is a constant $C(r)$ independent of $R$ such that for all $R>r+1$,

$$
\sup _{B_{p}(r)}\left|\nabla u_{R}\right| \leq C(r)
$$

So, we have

$$
\underset{B_{p}(r)}{\operatorname{Osc}} u_{R} \leq 2 r C(r)
$$

for all $R>r+1$. Suppose $\inf _{\partial B_{p}\left(r_{1}\right)} u_{R} \leq 0$ for all $R>r_{1}$; then $u_{R}(p) \leq 2 r_{1} C\left(r_{1}\right)$ by (1.4) with $r=r_{1}$. Therefore we may assume that $\inf _{\partial B_{p}\left(r_{1}\right)} u_{R} \geq 0$. In this 
case, $u_{R} \geq 0$ on $B_{p}(R) \backslash B_{p}\left(r_{1}\right)$, by the maximum principle, (1.1) and the fact that $f$ is subharmonic outside $B_{p}\left(r_{0}\right)$. Hence we have

$$
\begin{aligned}
0 & \geq \int_{B_{p}(R) \backslash B_{p}\left(r_{1}\right)} u_{R} \Delta u_{R} \\
& =-\int_{B_{p}(R) \backslash B_{p}\left(r_{1}\right)}\left|\nabla u_{R}\right|^{2}-\int_{\partial B_{p}\left(r_{1}\right)} u_{R} \frac{\partial u_{R}}{\partial r} \\
& =-\int_{B_{p}(R) \backslash B_{p}\left(r_{1}\right)}\left|\nabla u_{R}\right|^{2}-\int_{\partial B_{p}\left(r_{1}\right)}\left(u_{R}-u_{R}(p)\right) \frac{\partial u_{R}}{\partial r}-u_{R}(p) \int_{\partial B_{p}\left(r_{1}\right)} \frac{\partial u_{R}}{\partial r},
\end{aligned}
$$

where we have used the fact that $u_{R}=0$ on $\partial B_{p}(R)$. By (1.1), we have

$$
\int_{\partial B_{p}\left(r_{1}\right)} \frac{\partial u_{R}}{\partial r}=-\int_{\partial B_{p}\left(r_{1}\right)} \frac{\partial f}{\partial r} .
$$

Combining this with (1.5), we have

$$
u_{R}(p) \int_{\partial B_{p}\left(r_{1}\right)} \frac{\partial f}{\partial r} \leq \int_{B_{p}(R) \backslash B_{p}\left(r_{1}\right)}\left|\nabla u_{R}\right|^{2}+\int_{\partial B_{p}\left(r_{1}\right)}\left(u_{R}-u_{R}(p)\right) \frac{\partial u_{R}}{\partial r} .
$$

By (1.3), (1.4) and the fact that $\int_{\partial B_{p}\left(r_{1}\right)} \frac{\partial f}{\partial r}>0$, the lemma follows.

Theorem 1.2. Let $M$ be a complete noncompact manifold. Then the following are equivalent:

(a) Every harmonic function on $M$ with finite Dirichlet integral is bounded.

(b) Every nonnegative smooth function on $M$ with finite Dirichlet integral which is subharmonic outside a compact set is bounded.

Proof. To prove that (b) implies (a), let $f$ be a harmonic function with finite Dirichlet integral; then $g=\sqrt{f^{2}+1}$ is subharmonic with finite Dirichlet integral. Therefore by (b), $g$ and hence $f$ are bounded.

To prove that (a) implies (b), let $f \geq 0$ be a smooth function with finite Dirichlet integral which is subharmonic outside a compact set. For simplicity, let us assume that $f$ is subharmonic on $M \backslash B_{p}(1)$, where $p$ is a fixed point in $M$. Consider the function $\phi=\left(1+f^{2}\right)^{\frac{1}{2}}$. It is easy to see that $\phi$ has finite Dirichlet integral and that $\phi$ is bounded if and only if $f$ is bounded. Since $f$ is subharmonic outside $B_{p}(1)$ and $f \geq 0$, direct computations show

$$
\Delta \phi \geq \frac{|\nabla f|^{2}}{\left(1+f^{2}\right)^{\frac{3}{2}}}
$$

on $M \backslash B_{p}(1)$. In particular, $\phi$ is subharmonic on $M \backslash B_{p}(1)$. Given any $r>1$, if $|\nabla f| \equiv 0$ on $M \backslash B_{p}(r)$, then $f$ must be constant on each component of $M \backslash \overline{B_{p}(r)}$. By the maximum principle, we have

$$
\sup _{M \backslash B_{p}(r)} f=\sup _{\partial B_{p}(r)} f .
$$


Hence, $f$ must be bounded and we are done. So we may assume that for all $r>1$ there is $R>r$ such that $\int_{B_{p}(R) \backslash \overline{B_{p}(r)}} \frac{|\nabla f|^{2}}{\left(1+f^{2}\right)^{\frac{3}{2}}}>0$. By (1.6), and by replacing $f$ by $\phi$, we may assume that for all $r>1$ there is $R>r$ such that

$$
\int_{B_{p}(R) \backslash \overline{B_{p}(r)}} \Delta f>0 .
$$

For $R>1$, let $u_{R}$ be the solution of $\Delta u_{R}=-\Delta f$ on $B_{p}(R)$ and $u_{R}=0$ on $\partial B_{p}(R)$. We want to show that $u_{R}$ are uniformly bounded on compact sets. By the maximum principle, $u_{R}+f$ is nonnegative, since $f \geq 0$. Hence $u_{R}$ are uniformly bounded from below on compact sets. If there is $R_{0}>1$ such that $\inf _{\partial B_{p}(1)} u_{R_{0}} \geq 0$, then $u_{R}+f \geq f$ on $B_{p}\left(R_{0}\right) \backslash B_{p}(1)$. Therefore

$$
\begin{aligned}
0 & =\int_{\partial B_{p}\left(R_{0}\right)} \frac{\partial}{\partial r}\left(u_{R_{0}}+f\right) \\
& \leq \int_{\partial B_{p}\left(R_{0}\right)} \frac{\partial f}{\partial r}
\end{aligned}
$$

where we have used the fact that $\Delta\left(u_{R}+f\right)=0$ on $B_{p}\left(R_{0}\right)$ and $u_{R_{0}}=0$ on $\partial B_{p}\left(R_{0}\right)$. By (1.7), there is $R_{1}>R_{0}$, such that $\int_{B_{p}\left(R_{1}\right) \backslash B_{p}\left(R_{0}\right)} \Delta f>0$. Therefore by (1.6) and (1.8) we have

$$
\begin{aligned}
\int_{\partial B_{p}\left(R_{1}\right)} \frac{\partial f}{\partial r} & =\int_{B_{p}\left(R_{1}\right) \backslash B_{p}\left(R_{0}\right)} \Delta f+\int_{\partial B_{p}\left(R_{0}\right)} \frac{\partial f}{\partial r} \\
& >0 .
\end{aligned}
$$

By Lemma 1.1, $u_{R}(p)$ are uniformly bounded from above.

If $\inf _{\partial B_{p}(1)} u_{R} \leq 0$ for all $R$, then arguing as in the proof of Lemma 1.1, $u_{R}(p)$ are also uniformly bounded from above. Hence as in the proof of Lemma 1.1, one can show that $u_{R}$ are uniformly bounded on compact sets, and there is a subsequence of $u_{R}+f$ which converges to a harmonic function $h$ on $M$ with finite Dirichlet integral. By assumption, $h$ is bounded. It is easy to see from this that $f$ is also bounded from above.

Corollary 1.3. Let $M$ be a complete noncompact manifold satisfying one of the equivalent conditions of Theorem 1.2. Let $f \geq 0$ be a function which is subharmonic outside a compact set on $M$ such that $\int_{B_{p}(R)} f^{2} \leq C R^{2}$ for some point $p \in M$, for some constant $C$ for all $R \geq 1$; then $f$ is bounded.

Proof. Using the standard cutoff function argument, one can show that $f$ has finite Dirichlet integral.

Theorem 1.2 can also be proved by using potential theory as noted by Terry Lyons [Ly]. Using our method of proof of Theorem 1.2, we can obtain a new criterion for a complete noncompact manifold to have a positive Green's function.

Theorem 1.4. Let $M$ be a complete noncompact manifold. Then $M$ has a positive Green's function if and only if $M$ has a nonnegative nonconstant subharmonic function with finite Dirichlet integral.

Proof. Suppose $M$ has a positive Green's function and we use $G(x, y)$ to denote the minimal positive Green's function. Let $f$ be a smooth nonnegative function with 
support in $B_{p}(1)$, where $p$ is some fixed point in $M$ such that $f$ is not identically equal to 0 . Then

$$
u(x)=-\int_{M} G(x, y) f(y) d y
$$

is a well-defined smooth function such that $\Delta u=f$ in $M$. In particular, $u$ is a nonconstant subharmonic function since $f$ is nonnegative and not identically equal to zero. On $M \backslash B_{p}(1), u$ is harmonic and equal to $\lim _{R \rightarrow \infty} u_{R}$ where $u_{R}$ is the harmonic function on $B_{p}(R) \backslash B_{p}(1)$ for $R>1$ such that $u_{R}=u$ on $\partial B_{p}(1)$ and $u_{R}=0$ on $\partial B_{p}(R)$. It is easy to see that the Dirichlet integral of $u_{R}$ is uniformly bounded. This implies that $u$ has finite Dirichlet integral. It is also easy to see that $u$ is bounded. Hence $M$ has a nonnegative nonconstant subharmonic function with finite Dirichlet integral.

To prove the converse, suppose $M$ has a nonnegative nonconstant subharmonic function $f$ with finite Dirichlet integral. If $\Delta f \equiv 0$, then by a theorem of [S-S-G], $M$ will have a nonconstant bounded harmonic function with finite Dirichlet integral. By a well-known result, $M$ has a positive Green's function, see for example [L-T 1]. Therefore without loss of generality, we may assume that $\Delta f(x) \geq \epsilon$ on $B_{p}(a)$ for some $a>0$ and some $\epsilon>0$. For $R>a$, let

$$
h_{R}(x)=\int_{B_{p}(R)} G_{R}(x, y) \Delta f(y) d y,
$$

on $B_{p}(R)$, where $G_{R}(x, y)$ is the positive Green's function on $B_{p}(R)$ with zero boundary data. Then $\Delta h_{R}=-\Delta f$ on $B_{p}(R)$ and $h_{R}=0$ on $\partial B_{p}(R)$. By Lemma $1.1, h_{R}(p)$ is uniformly bounded from above, therefore there is a constant $C$ independent of $R$ such that

$$
\begin{aligned}
C_{4} & \geq \int_{B_{p}(R)} G_{R}(p, y) \Delta f(y) d y \\
& \geq \epsilon\left(\min _{y \in B_{p}(a) \backslash B_{p}\left(\frac{a}{2}\right)} G_{R}(p, y)\right)\left(V_{p}(a)-V_{p}\left(\frac{a}{2}\right)\right),
\end{aligned}
$$

where we have used the fact that $\Delta f \geq 0, \Delta f \geq \epsilon$ on $B_{p}(a)$ and $G_{R}>0$. Hence there is a constant $C_{5}$ independent of $R$ such that

$$
\min _{y \in B_{p}(a) \backslash B_{p}\left(\frac{a}{2}\right)} G_{R}(p, y) \leq C_{5}
$$

By the results in [L-T 1], we conclude that $M$ has a positive Green's function.

\section{A CLASS OF MANIFOLDS}

In this section, we will discuss some sufficient conditions for a manifold to satisfy the condition that every harmonic function with finite Dirichlet integral on the manifold is bounded. To simplify our statements, we will say that such a manifold satisfies condition (A). 
Proposition 2.1. Let $M$ be a complete noncompact manifold with no nonconstant bounded harmonic function. Then every harmonic function on $M$ with finite Dirichlet integral is constant. In particular $M$ satisfies (A).

Proof. Let $f$ be a harmonic function on $M$ with finite Dirichlet integral. Suppose $f$ is nonconstant; then by a theorem in [S-S-G], there is a nonconstant bounded harmonic function on $M$ with finite Dirichlet integral. This is a contradiction since $M$ has no nonconstant bounded harmonic function.

It is well known that if $M$ has nonnegative Ricci curvature, then $M$ has no nonconstant bounded harmonic function by a theorem in $[\mathrm{Y}]$. By $[\mathrm{Gr}]$ and $[\mathrm{SC}]$, if $M$ is quasi-isometric to a complete noncompact manifold with nonnegative Ricci curvature, then $M$ also has no nonconstant bounded harmonic function. Hence all these kinds of manifolds satisfy (A). In fact, in [Gr], a complete noncompact manifold $M$ which satisfies the following conditions are considered:

(I) There exists a constant $A>0$ such that for any $x \in M$ and for all $R>0$

$$
V_{x}(2 R) \leq A V_{x}(R)
$$

where $V_{x}(r)$ is the volume of the geodesic ball $B_{x}(r)$ of radius $r$ centered at $x$.

(II) There exist constants $N>1$ and $a>0$ such that for any $f \in C^{\infty}\left(B_{x}(N R)\right)$

$$
\frac{a}{R^{2}} \inf _{\alpha \in \mathbb{R}} \int_{B_{x}(R)}(f-\alpha)^{2} \leq \int_{B_{x}(N R)}|\nabla f|^{2} .
$$

Note that (I) and (II) are invariant under quasi-isometry. This class of manifolds includes complete noncompact manifolds with nonnegative Ricci curvature. It was proved in $[\mathrm{Gr}]$ that a complete noncompact manifold satisfying (I) and (II) has no nonconstant bounded harmonic function, and hence it also satisfies (A).

Proposition 2.2. Let $M_{1}$ and $M_{2}$ be two n-dimensional manifolds satisfying (A). Then the connected sum of $M_{1}$ and $M_{2}$ also satisfies (A).

Proof. Let $M$ be the connected sum of $M_{1}$ and $M_{2}$. Let $f$ be a harmonic function on $M$ with finite Dirichlet integral. Then $f$ is defined and harmonic outside a compact set of $M_{1}$. Therefore $\sqrt{f^{2}+1} \geq 0$ is subharmonic outside a compact set of $M_{1}$. Since $f$ has finite Dirichlet integral, $f$ is bounded near infinity of $M_{1}$ by Theorem 1.2. Similarly, $f$ is bounded near infinity of $M_{2}$. Therefore $f$ is bounded.

Before we state our next result, we introduce some terminology. Let $M$ be a complete noncompact manifold and $K$ be a compact subset of $M$. An unbounded component of $M \backslash K$ is called an end of $M$ with respect to $K$. Let $p$ be a fixed point in $M$. Suppose the number of unbounded components of $M \backslash \overline{B_{p}(R)}$ is bounded by a constant independent of $R$, for all $R>0$; then $M$ is said to have finitely many ends. In this case, an end $E$ of $M$ is an unbounded component of $M \backslash \overline{B_{p}\left(R_{0}\right)}$ for some fixed $R_{0}$ such that the number of unbounded components of $M \backslash \overline{B_{p}(R)}$ is equal to the number of unbounded components of $M \backslash \overline{B_{p}\left(R_{0}\right)}$ for all $R \geq R_{0}$. An end $E$ of $M$ with respect to some compact set $K$ is said to be large if $\int_{a}^{\infty} t / V_{E}(t) d t<\infty$ for some $a>0$ where $V_{E}(t)$ is the volume of $B_{p}(t) \cap E$. Otherwise the end $E$ is said to be small. An end $E$ is said to satisfy the volume comparison condition (VC) if there is $\zeta>0$ and $r>0$ such that for all $R>r, V_{E}(R) \leq \zeta V_{x}(R / 2)$ for all $x \in \partial B_{p}(R) \cap E$. Manifolds with finitely many ends, each of which satisfies (VC), have been studied by $\mathrm{Li}$ and the second author [L-T 3]. We have the following: 
Proposition 2.3. Let $M$ be a complete noncompact manifold and $p$ be a fixed point in $M$. Suppose the Ricci curvature of $M$ satisfies $\operatorname{Ric}_{M}(x) \geq-C(1+r(x))^{-2}$ for some constant $C>0$, for all $x$, where $r(x)$ is the distance from $x$ to $p$. Suppose $M$ has only finitely many ends and each large end satisfies condition (VC). Then every harmonic function with finite Dirichlet integral on $M$ must be bounded.

Proof. Let $f$ be a harmonic function on $M$ with finite Dirichlet integral. It is sufficient to prove that $f$ is bounded on each end. Let $E$ be an end and $M_{E}$ be the double of $E$. Then $f$ can be considered as a function on $M_{E}$ which is harmonic outside a compact set with finite Dirichlet integral. Suppose that $E$ is small; then $M_{E}$ has no positive Green's function (see [L-T 1] for example). In particular, $M_{E}$ has no nonconstant bounded harmonic function. By Proposition 2.1 and Theorem 1.2 , we can conclude that $f$ is bounded on $M_{E}$. Suppose that $E$ is large. Since $E$ satisfies (VC), by the proof of Lemma 2.3 in [L-T 3], we can also conclude that $f$ is bounded on $M_{E}$. This completes the proof of the theorem.

Corollary 2.4. Let $M$ be a complete noncompact manifold and $p$ be a fixed point. Suppose the sectional curvature of $M$ satisfies $K_{M}(x) \geq-\lambda(r(x))$ where $r(x)$ is the distance from $p$ to $x$ and $\lambda:[0, \infty) \rightarrow[0, \infty)$ is a nonincreasing function such that $\int_{0}^{\infty} t \lambda(t) d t<\infty$. Then $M$ satisfies (A).

Proof. It was proved in $[\mathrm{K}]$ that $M$ has finitely many ends. It is easy to see that the Ricci curvature of $M$ satisfies the condition in Proposition 2.3. It was also proved in [L-T 3] that each end of $M$ satisfies the volume comparison condition (VC). Hence by Proposition 2.3, $M$ satisfies (A).

\section{Applichtions to harmonic maps}

In this section, we will apply the previous results to study the uniqueness problem for harmonic maps on complete noncompact manifolds. First, let us prove a general theorem. Recall that a Cartan-Hadamard manifold is a simply connected complete manifold with nonpositive sectional curvature.

Theorem 3.1. Let $M$ be a complete noncompact manifold satisfying condition (A), i.e., every harmonic function with finite Dirichlet integral is bounded. Let $N$ be a Cartan-Hadamard manifold. Then any harmonic map from $M$ into $N$ with finite total energy must have bounded image.

Proof. Let $u: M \rightarrow N$ be a harmonic map with finite total energy. Let $y_{0}$ be a fixed point in $N$ and let $r(y)$ be the distance function on $N$ from $y_{0}$. Then it is well known that $f=\sqrt{(r \circ u)^{2}+1}$ is subharmonic on $M$ (see [S-Y 2], for example). Since $u$ has finite total energy, it is easy to see that $f$ has finite Dirichlet integral. Since $M$ satisfies (A), by Theorem 1.2, $f$ is bounded, and hence $u(M)$ is bounded.

Let $M$ be a complete noncompact manifold. Assume that there is a bounded domain $D$ of $M$, such that every bounded harmonic function on $M$ is asymptotically constant near infinity at each unbounded component of $M \backslash \bar{D}$. Note that $M$ supports no nonconstant bounded harmonic function if and only if $M$ satisfies the above assumption with $D$ to be the empty set. Liouville type results on bounded harmonic maps from such a manifold into a Cartan-Hadamard manifold have been studied by Sung, Wang and the second author [S-T-W]. Using Theorem 3.1 and the results in $[\mathrm{S}-\mathrm{T}-\mathrm{W}]$, we have: 
Theorem 3.2. Let $M$ be a complete noncompact manifold satisfying condition (A) and the above assumptions with some bounded domain D. Let $N$ be a CartanHadamard manifold. Then every harmonic map with finite total energy from $M$ into $N$ is asymptotically constant near infinity at each unbounded component of $M \backslash \bar{D}$. In particular, if $M$ supports no nonconstant bounded harmonic function, then any harmonic map with finite total energy from $M$ into $N$ must be constant.

Proof. The theorem follows from Theorem 3.1, Proposition 2.1 and the results in $[\mathrm{S}-\mathrm{T}-\mathrm{W}]$.

For the case when $M$ has nonnegative Ricci curvature, the last statement of the theorem was proved by Schoen and Yau [S-Y 1]. In that case, the assumption that $N$ is simply connected can be dropped. The last statement for the case when $M$ is quasi-isometric to a complete noncompact manifold with nonnegative Ricci curvature was also proved by Wang [W] using other methods.

Next, we will study the question of the uniqueness of homotopic maps on noncompact manifolds into manifolds with nonpositive sectional curvature. Let $M$ be a complete noncompact manifold and $N$ be a complete manifold with nonpositive curvature. Let $u$ and $v$ be two homotopic maps from $M$ into $N$. Using the setup in [S-Y 2], let $\tilde{M}$ and $\tilde{N}$ be the universal covers of $M$ and $N$ respectively. Let $F:[0,1] \times M \rightarrow N$ be a homotopy of $u$ and $v$ such that $F(0, x)=u(x)$ and $F(1, x)=v(x)$. Choose a lifting $\tilde{F}:[0,1] \times \tilde{M} \rightarrow \tilde{N}$ and let $\tilde{u}(\tilde{x})=\tilde{F}(0, \tilde{x})$ and $\tilde{v}(\tilde{x})=\tilde{F}(1, \tilde{x})$. Then $\tilde{u}$ and $\tilde{v}$ are liftings of $u$ and $v$ respectively. Define $\rho_{u, v}(x)=\operatorname{dist}_{\tilde{N}}(\tilde{u}(\tilde{x}), \tilde{v}(\tilde{x}))$, for $x \in M$, where $\tilde{x} \in \tilde{M}$ is a point over $x$. It is easy to see that $\rho_{u, v}$ is well defined. Since $N$ has nonpositive curvature, $\rho_{u, v}^{2}$ is smooth on $M$ provided $u$ and $v$ are smooth. Note that $\rho_{u, v}(x)$ is the length of the minimal goedesic in $N$ which is in the homotopic class of the curve $F(t, x), 0 \leq t \leq 1 . \rho_{u, v}$ may not be bounded even if $N$ is compact. Under this setting, we have:

Theorem 3.3. Let $M$ be a complete noncompact manifold satisfying condition (A), that is, every harmonic function on $M$ with finite Dirichlet integral is bounded. Let $N$ be a complete manifold with nonpositive curvature. Let $u$ and $v$ be two homotopic harmonic maps from $M$ into $N$ with finite total energy. Then $\rho_{u, v}$ is bounded. Furthermore, if the universal cover $\tilde{M}$ has no nonconstant bounded harmonic functions and $v$ is a constant, then $u$ must be a constant.

Proof. It is known that $f=\sqrt{\rho_{u, v}^{2}+1}$ is subharmonic with finite total energy (see [S-Y 2]). By Theorem 1.2, we conclude that $f$ is bounded. Suppose $\tilde{M}$ has no nonconstant bounded harmonic function, and that $v$ is constant. Then the lifting $\tilde{v}$ obtained above is also a constant map. Here we have assumed that $\tilde{M}$ is connected. Hence the image of $\tilde{u}$ is bounded and it must be a constant map by [Ke]. Therefore, $u$ is also a constant map.

Corollary 3.4. Let $M$ be a complete noncompact manifold which is quasi-isometric to a complete noncompact manifold with nonnegative Ricci curavture. Let $N$ be a complete manifold with nonpositive curvature (which may not be simply connected). Every harmonic map with finite total energy from $M$ into $N$ which is homotopic to constant must be constant.

One can also generalize some uniqueness theorems of Schoen and Yau [S-Y 2] on homotopic harmonic maps with finite total energy from a complete manifold $M$ 
with finite volume into a complete manifold with nonpositive or strictly negative sectional curvature. We would like to relax the condition that $M$ has finite volume by only assuming that $M$ is parabolic. That is, $M$ has no positive Green's function. Note that a complete noncompact manifold with finite volume is always parabolic (see for example [V] or [L-T1]). Suppose $M$ is parabolic, in particular $M$ has no nonconstant bounded harmonic function. Then $M$ satisfies condition (A) by Proposition 2.1. Let $u$ and $v$ be two homotopic harmonic maps with finite total energy into a complete manifold with nonpositive sectional curvature. Using previous notation in Theorem 3.3, $f=\sqrt{\rho_{u, v}^{2}+1}$ is bounded by that theorem. Since $f$ is subharmonic and $M$ is parabolic, $f$ must be constant. Hence $\rho_{u, v}$ is constant. Therefore, the methods in [S-Y 2] can be carried over to conclude that:

Theorem 3.5. Suppose $M$ is a complete noncompact parabolic manifold and $N$ is a complete manifold with nonpositive sectional curvature. Let $u$ and $v$ be homotopic harmonic maps with finite total energy from $M$ into $N$. Then there is a smooth one-parameter family of harmonic maps $f_{t}: M \rightarrow N, 0 \leq t \leq 1$, with $f_{0}=u$, $f_{1}=v$, such that $f_{t}(x)$ is a geodesic of constant speed (independent of $x$ ) for all $x$.

Theorem 3.6. Suppose $M$ is a complete noncompact parabolic manifold and $N$ is a complete manifold with strictly negative sectional curvature. Let $u$ be a harmonic map from $M$ into $N$ with finite total energy. There is no other harmonic map of finite energy which is homotopic to u unless $u(M)$ is contained in a geodesic of $N$.

\section{ACKNOWLEDGMENTS}

The second author would like to thank Terry Lyons for some useful discussions on Theorem 1.2. The authors would also like to thank Peter Li and Jiaping Wang for their interest in the work. The work was done while the second author was visiting the Chinese University of Hong Kong. He would like to express his gratitude for their hospitality.

\section{REFERENCES}

[Cg] S. Y. Cheng, Liouville theorem for harmonic maps, Proc. Sympos. Pure Math., vol. 36, Amer. Math. Soc., Providence, RI, 1980, pp. 147-151. MR 81i:58021

[Ci] H. I. Choi, On the Liouville theorem for harmonic maps, Proc. Amer. Math. Soc. 85 (1982), 91-94. MR 83j:53073

[G] A. A. Grigor'yan, The heat equation on noncompact Riemannian manifolds, Mat. Sb. 182 (1) (1991), 55-87; English transl., Math. USSR-Sb. 72 (1992), no. 1, 47-77. MR 92h:58189

[K] A. Kasue, A compactification of a manifold with asymptotically nonnegative curvature, Ann. Sci. École Norm. Sup. (4) 21 (1988), 593-622. MR 90d:53049

[Ke] W. S. Kendall, Probability, convexity, and harmonic maps with small image I: uniqueness and fine existence, Proc. London Math. Soc. (3) 61 (1990), 371-406. MR 91g:58062

[L-T 1] P. Li and L. F. Tam, Symmetric Green's functions on complete manifolds, Amer. J. Math. 109 (1987), 1129-1154. MR 89f:58129

[L-T 2] The heat equation and harmonic maps of complete manifolds, Invent. Math. 105 (1991), 1-46. MR 93e:58039

[L-T 3] , Green's functions, harmonic functions and volume comparison, J. Differential Geom. 41 (1995), 277-318.

[Ly] T. J. Lyons, private communication.

[SC] L. Saloff-Coste, Uniformly elliptic operators on Riemannian manifolds, J. Differential Geom. 36 (1992), 417-450. MR 93m:58122 
[S-S-G] L. Sario, M. Schiffer, and M. Glasner, The span and principal functions in Riemannian spaces, J. Analyse Math. 15 (1965), 115-134. MR 32:1655

[S-Y 1] R. Schoen and S. T. Yau, Harmonic maps and the topology of stable hypersurfaces and manifolds of nonnegative Ricci curvature, Comment. Math. Helv. 39 (1976), 333-341. MR 55:11302

[S-Y 2] Compact group actions and the topology of manifolds with non-positive curvature, Topology 18 (1979), 361-380. MR 81a:53044

[S-T-W] J.-T. Sung, L.-F. Tam, and J.-P. Wang, Bounded harmonic maps on a class of manifolds, Proc. Amer. Math. Soc. (to appear).

[V] N. Varopoulos, Potential theory and diffusion on Riemannian manifolds, Conference on Harmonic Analysis in Honor of Antoni Zygmund, Vols. I, II, Wadsworth Math. Ser., Wadsworth, Belmont, CA, 1983, pp. 821-837. MR 85a:58103

[W] J.-P. Wang, private communication.

[Y] S. T. Yau, Harmonic functions on complete Riemannian manifolds, Comm. Pure Appl. Math. 28 (1975), 201-228. MR 55:4042

Department of Mathematics, University of California, los Angeles, California 90024 and Department of Mathematics, The Chinese University of Hong Kong, Hong KONG

Department of Mathematics, University of California, Irvine, California 92717-3875 Current address: Department of Mathematics, The Chinese University of Hong Kong, Shatin, Hong Kong

E-mail address: Itam@math.uci.edu

Department of Mathematics, The Chinese University of Hong Kong, Shatin, Hong KONG

E-mail address: tomwan@cuhk.hk 